Canadian University Music Review

Revue de musique des universités canadiennes

\title{
Pour une analyse structurale des Variations opus 27 de Webern
}

\section{Edmond Costère}

Numéro 7, 1986

URI : https://id.erudit.org/iderudit/1014089ar

DOI : https://doi.org/10.7202/1014089ar

Aller au sommaire du numéro

Éditeur(s)

Canadian University Music Society / Société de musique des universités

canadiennes

ISSN

0710-0353 (imprimé)

2291-2436 (numérique)

Découvrir la revue

Citer cet article

Costère, E. (1986). Pour une analyse structurale des Variations opus 27 de

Webern. Canadian University Music Review / Revue de musique des universités

canadiennes, (7), 103-126. https://doi.org/10.7202/1014089ar

All Rights Reserved (C Canadian University Music Society / Société de musique des universités canadiennes, 1986
Ce document est protégé par la loi sur le droit d'auteur. L'utilisation des services d'Érudit (y compris la reproduction) est assujettie à sa politique d'utilisation que vous pouvez consulter en ligne.

https://apropos.erudit.org/fr/usagers/politique-dutilisation/ 


\title{
POUR UNE ANALYSE STRUCTURALE DES VARIATIONS OPUS 27 DE WEBERN
}

\author{
Edmond Costère
}

\section{Schoenberg et les affinités sonores}

La traduction du Traité d'Harmonie de Schoenberg (1983) permet désormais au lecteur de langue française de mieux approfondir la pensée du compositeur-théoricien.

Il y pose ce principe essentiel qui domine tout son exposé : l'harmonie consiste en "un équilibre de toutes les forces de tension possibles" (1983 : 54), lesquelles s'exercent selon "la loi du plus court chemin" (ibidem : 64, 158).

Limitant d'abord son enseignement à la musique tonale, il fait état, d'une part des attractions primordiales que les hauteurs doivent aux propensions naturelles des harmoniques, mettant ainsi en évidence les "forces attractives" de quinte et de quarte (ibidem : 38, 43, 179), et, d'autre part, des tensions chromatiques propres au phénomène naturel du glissement sonore, dont il place "les enchaînements de seconde ascendante et de seconde descendante" sur le même plan que ceux de quinte et de quarte (ibidem : 259).

Débordant ensuite le diatonisme, l'examen des moyens de "suspendre la tonalité" le conduit, page 514 , à cette réflexion, maintes fois citée à propos du dodécaphonisme, sur les successions de deux accords combinant la totalité ou la quasi-totalité des douze sons : 
Ces dernières semblent réglées par une tendance selon laquelle le second accord de l'enchaînement recueille les sons qui font défaut au premier et qui sont généralement situés un demi-ton plus haut ou plus bas que les sons du premier accord,

et il souligne la persistance de ces affinités même après reports d'octave.

Mais c'est dans Der Mondfleck de son Pierrot lunaire, un an après la publication, en 1911, de son Traité d'Harmonie, qu'il formule le mieux sa pensée, en qualifiant de "quasi kadenzierend" la propension, sur un MIb, de l'ensemble des sons MI, SIb, LAb et RE.

Par cette assimilation, à une cadence, de l'aboutissement conjugué des mouvements naturels de demi-ton, de quarte et de quinte, s'accomplit, selon les directives mêmes de sa "loi du plus court chemin", cet "équilibre de toutes les forces de tension possibles" dont il entendait animer les hauteurs.

Et ce même équilibre d'affinités, il l'a mis plus tard en oeuvre dans "les accords-types" de son "Ecole de Vienne", qui associent à un son principal deux, ou même trois, des sons distants de ces mêmes intervalles de quinte, de quarte et de demi-ton, destinés à le prolonger, à l'exemple de la tierce et de la quinte prolongeant le son fondamental de l'accord parfait. ${ }^{1}$

1 Dans ses Variations, opus 27, Webern use constamment de ces accordstypes, en joignant à un son principal, dans un même accord, soit sa quinte et sa seconde mineure descendante : mesure 16 du premier mouvement ; soit sa quinte et sa second mineure ascendante : mesures $9,16,45,52$ et 54 du ler mouvement, mesures 3,8 et 15 du 2 e mouvement, mesures 24,31 , $34,37,39,60,62,65$ du 3e mouvement ; soit sa quarte et sa seconde mineure descendante : mesures $8,10,16,18,44,46$ et 52 du ler mouvement, mesures $4,9,15$ et 19 du $2 \mathrm{e}$ mouvement et mesures $26,28,33,42,56,58$ et $63 \mathrm{du} 3 \mathrm{e}$ mouvement ; soit ses deux secondes mineures et sa quinte : mesures 16 du ler mouvement et $34,37,39$ et 41 du 3e mouvement ; soit ses deux secondes mineures et sa quarte : mesures 52 du ler mouvement et 42 du 3e mouvement ; soit seulement ses deux secondes mineures : mesures $11,14,47$ et 50 du premier mouvement. 
Pourquoi privilégier à ce point ces intervalles ? C'est qu'ils procèdent des phénomènes acoustiques élémentaires dont notre organe pharyngobuccal est lui-même le siège ; propensions naturelles des harmoniques avec ses intervalles premiers d'octave et de quinte, glissements naturels des sons chromatiques, avec les demi-tons de notre échelle usuelle.

Grâce à la spontanéité innée de ces inflexions, une sorte de certitude sonore s'exprime dans l'aboutissement sur sa fin de l'intervalle d'octave, de l'intervalle de quinte, de l'intervalle de demi-ton. Et l'émission successive des deux sons qui emplissent chacun d'eux nous est tellement consubstantielle, que notre entendement la perçoit comme une évidence, l'évidence propre à ce qui se résoud sur son achèvement le plus naturel, comme un affermissement, une affirmation ou une conclusion.

Au surplus, l'affinité réciproque qui unit les deux sons de ces intervalles s'exerce en montant comme en descendant, de telle sorte qu'à l'octave, la quinte et le demi-ton, s'ajoute ici l'intervalle de quarte.

Une telle prépondérance fonctionnelle de ces quatre intervalles est loin d'être arbitraire, puisqu'elle relève d'un processus acoustico-psychique. Et le principe même de leurs propensions avait en fait si bien dirigé les mouvements obligés de notre harmonie traditionnelle, qu'elles en constituent maintenant la justification rationnelle la plus convaincante.

\section{Vocation des affinités naturelles}

A l'instar de cette tradition, par ces mouvements résolutifs d'octave, de quinte, de quarte et de demi-ton, toute figure musicale devient apte à tendre vers le son ou les sons qui la closent, pour peu qu'elle contienne tout ou une partie des notes situées à distance d'octave, de quinte, de quarte et de demi-ton de ceux-ci, en montant comme en descendant. ${ }^{2}$

mouvement ; soit seulement ses deux secondes mineures : mesures 11,14 , 47 et 50 du premier mouvement.

2 Tels les sons de la cadence traditionnelle de SOL-SI-RE-FA sur DO-MI-SOL, 
Et la propension est d'autant plus efficace que ces notes y sont en plus grand nombre, qu'elles y ressortent davantage, et que leur tessiture se rapproche le plus du mouvement originel de leur affinité. En revanche, leur éloignement du son ou des sons ultimes, leur petit nombre, leur insignifiance, ou des reports d'octave, ne peuvent que plus ou moins compromettre le pouvoir cadentiel de l'ensemble.

Si cette logique des hauteurs prévaut, l'oeuvre la plus contraire à l'harmonie traditionnelle s'éclaire au fur et à mesure qu'on y décèle les propensions latentes, de la manière même dont les signes de ponctuation viennent restituer l'intégralité de son sens à un texte qui aurait été dépouillé de ces signes, avec toutes les nuances expressives qu'auparavant lui avaient valu virgules, points, point-virgules, points de suspension, d'interrogation ou d'exclamation.

Ces tensions réciproques des affinités d'octave, de quinte, de quarte et de demi-ton confèrent leur pouvoir dialectique à toutes les hauteurs qui gravitent à l'intérieur d'une échelle tempérée comportant les trois premiers harmoniques, et peuvent y animer les oeuvres les plus opposées à la tradition, comme je l'ai montré ailleurs (Costère 1954, 1962). ${ }^{3}$

Tel est le cas des oeuvres atonales ou sérielles des trois maîtres de l'Ecole de Vienne. Pourtant, Schoenberg, Berg et Webern se sont gardés d'en révéler les procédés, au point de provoquer un contre-sens généralisé sur la compréhension de leurs oeuvres, car seule s'est imposée la connaissance des mécanisations sérielles.

grâce aux affinités naturelles vers le DO du SOL, du SI et du FA, vers le MI du SI et du FA, vers le SOL du SOL et du RE, affinités dont le nombre total surpasse celui de toutes les autres combinaisons, sans exception aucune, de quatre des sept sons DO, RE, MI, FA, SOL, LA et SI.

3 En ce qui concerne les diverses échelles possibles, le premier de ces ouvrages traite de leurs caractéristiques (pages 35 et suivantes), et énumère notamment les échelles capables de contenir, avec une approximation de moins d'un comma, les intervalles tempérés correspondant aux premiers harmoniques naturels. 
Le véritable propagateur en langue française de cette technique, et son prophète très écouté, René Leibowitz, a eu le grand mérite de décrire minutieusement ceux de ses impératifs qui s'attachent aux imbrications mêmes de la série. Mais il réduisait à ceci les affinités entre ses sons numérotés de 1 à 12 : "Le son 2 n'est apparenté qu'aux sons 1 et 3 , le 3 ne l'est qu'aux sons 2 et 4, etc." (Leibowitz 1949 : 95).

C'est pourtant le démenti le plus évident - et le moins compris - qu'on découvre au passage parmi les nombreux textes de Schoenberg que Christiane de Lisle a réunis dans la traduction française de Le Style et l'Idée (1977). Non seulement Schoenberg prend-t-il soin d'y énoncer une conception rigoureusement opposée de la série, "dont l'intelligibilité en tant qu'idée musicale ne dépend pas de ce que ses sons sont émis l'un après l'autre" (1977 : 156), mais il insiste sur la nécessité, dans "la nouvelle musique", d'une véritable "pantonalité" (ibid. : 220).

Pour lui, en effet, "si des sons doivent fournir une progression logique et intelligible, il existe nécessairement entre eux des relations tonales" (ibid. : 159), totalement indépendantes de la réglementation sérielle (ibid. : $74,188,189$ ), et tellement "inhérentes à la nature des sons" (ibid. : 158,159 ) que "sa musique ne pourra pas prétendre à l'ordre, à la logique, à l'intelligibilité, tant qu'elle n'aura pas obéi à ces lois" (ibid. : 166).

Mais, sur l'énoncé et les conséquences de ces lois, il s'est catégoriquement refusé à s'expliquer davantage, s'agissant, disait-il, de "procédés techniques que chacun a intérêt à trouver tout seul et peut trouver tout seul" (ibid. : 162). Lorsque Thomas Mann s'est permis, dans son Doktor Faustus, une analyse de "la composition avec douze sons", il en a tourné l'exposé en dérision, disant qu'iil le tient de $\mathrm{M}$. Adorno, qui n'en sait pas plus lui-même que le peu que j'ai pu en dire à mes élèves". Et à cette occasion, il n'a pas hésité à poser en principe que "la vérité profonde restera une science secrète jusqu'à ce que quelqu'un soit illuminé par la vertu d'un don inné qu'il n'aura pas sollicité" (ibid. : 296). 
C’était donc de propos délibéré qu'il avait entendu réserver à ceux qui ont constitué avec lui l'Ecole de Vienne l'exclusivité des moyens constructifs propres à sa loi du plus court chemin. ${ }^{4}$

\section{Les Variations opus 27 de Webern}

C'est très exactement ce que confirme la dernière édition des Variations Opus 27 de Webern. 5

En y reproduisant les nombreuses recommandations de celui-ci pour leur interprétation, le pianiste Peter Stadlen qui, en 1937, a donné de cette oeuvre la première exécution publique, dénonce en effet le refus catégorique de Webern de lui révéler quoi que ce soit des structures fonctionnelles de sa mise en oeuvre. "A aucun moment des longues semaines de nos entretiens, dit-il, Webern n'a consenti à aborder le moindre élément de ce qui s'y réfère. Mieux encore, lorsque je l'interrogeais à leur sujet, il refusait obstinément d'en parler". 6

En revanche, cette édition abonde en indications nouvelles sur le toucher, le phrasé, le rythme, les fluctuations du tempo. Et les notations expressives que Stadlen a pris soin d'ajouter de mémoire tout au long

4 Lorsque, en 1954, j'ai posé cette loi comme le fondement de toute mon argumentation, je n'ai pas pu bénéficier de l'autorité de Schoenberg qui m'aurait été d'une particulière efficacité dans ce travail à contre-courant, car je ne connaissais pas sa propre formulation du même principe, ne disposant alors que des quelques passages de ses écrits, dont mon ami Max Deutsch consentait à me donner les traductions, telles que je les ai reproduites dans Lois et Styles des Harmonies musicales et dans Mort ou Transfigurations de l'Harmonie.

5 No 16 845, A.G. Universal Edition, Vienne (Autriche).

6 Page II, par. 8 du texte allemand et page V, par. 7 du texte anglais de 1"Introduction". Aucune confusion n'est possible ici avec les structurations sérielles, qui n'étaient alors, à Vienne, un secret pour personne dans le milieu musical. 
du texte musical, éveillent les séductions - insolites dans une oeuvre d'apparence tellement austère - de l'expressionnisme le plus intense, avec des formules telles que "lyrisme brûlant, tendresse, plainte secrète, rudesse, soupir haletant, élan passionné”.

Mais elle ne révèle rien de cette logique immanente, que Stadlen aurait tant souhaité connaître, dont voudrait prendre conscience tout exécutant soucieux d'aller au-delà du texte imprimé et de ses adjonctions afin d'en mieux pénétrer les arcanes, et dont Webern lui-même a dit qu'“il est impossible de les ignorer si l'on veut exprimer par des sons quelque chose qui ait un sens"7 (Webern 1980 : 158).

Pourtant, les procédés qui en découlent, il les a lui-même célés, se contentant d'énoncer que, loin de se limiter aux jeux de la série, sa musique "n'ignorait pas pour autant les lois inhérentes à la nature du son, notamment les rapports des harmoniques et de la fondamentale" (ibid. : 50 et 58), ainsi que "le besoin d'une note sensible" (ibid. : 73, $81,158)$, ce paradigme de l'affinité de glissement.

Mais l'ensemble de ces moyens transparaissent dans ses Variations Opus 27, pour peu qu'on s'avise d'en soumettre les hauteurs au test des affinités acoustico-psychiques en question, car ce sont très exactement les moyens discursifs de cette cinétique naturelle des sons qui dirigent l'intégralité du premier et du dernier mouvements de cette oeuvre.

Il s'agit d'abord des centres de gravité où convergent les affinités naturelles. Sans aucune exception, ils ponctuent toute interruption un peu importante du texte musical, dans l'édition primitive comme dans l'édition annotée. Que ce soit un arrêt passager, une mesure finale, un ritardando, une nouvelle variation ou un changement de tempo, Webern s'emploie toujours à souligner la fin de la figure arrêtée ou de la figure ultime, par la convergence, sur cette fin, de mouvements de quinte, de quarte ou de demi-ton, lui assurant ainsi cette impression

7 Les mots soulignés le sont par Webern. 
d'un aboutissement provisoire ou définitif, que la ponctuation confère aux phrases et aux membres de phrases d'un écrit.

Mieux encore, s'il répète un passage antérieur, ou s'il en renverse ou transpose les sons, toujours il provoque, par des reports d'octave, un renversement des potentiels d'affinité qui s'y trouvaient inclus, sans aucune exception.

Une si exacte coïncidence entre les points d'appui de l'écrit et les centres de gravité inhérents aux affinités confère à chaque suspension du phrasé une signification dialectique dont la logique sous-jacente vient compléter en profondeur les indications de Stadlen.

Mais ce n'est pas tout. En recourant aussi à la logique d'opposition du stable et de l'instable, Webern use de cet autre pouvoir des affinités naturelles : les différenciations qu'elles engendrent lorsqu'elles équilibrent ou déséquilibrent les figures qu'elles habitent.

En outre, l'intervalle de trois tons entiers, le triton, provoque un impact exactement contraire à celui des intervalles d'octave, de quinte, de quarte et de demi-ton. Au lieu de sembler s'achever sur le second des deux sons qu'il joint, il en cumule la propension vers d'autres sons, tels SI et FA qui tendent, l'un et l'autre, à la fois vers DO, MI, FA\# et SIb. Il en résulte qu'au coeur d'une figure qui aurait pu se résoudre sur un son donné, SI, par exemple, la présence de la note à distance de triton de celui-ci (un FA dans cette hypothèse) risque d'en contredire le potentiel d'aboutissement, puisqu'elle le déséquilibre. Et ici, également, cette emprise varie en fonction de l'emplacement et de l'importance de cette note désagrégatrice dans l'ensemble où elle se situe. 
Quant au second mouvement, par une autre manifestation de la dialectique de contraste, il échappe en son entier aux uns et aux autres de ces moyens dynamiques d'affinité 8 dont le premier et le troisième sont si abondamment pourvus (cf. exemple 1).

L'exécution qui peut se déduire de ces alternances de l'instable et du stable, de ces centres de gravité fonctionnels, de ces cadences, trouve son entière consécration - les renvois qui suivent en feront foi - dans les annotations de l'édition nouvelle. Mais elle va très au-delà, si elle en transcende les directives, grâce à cette cinétique latente, par laquelle Webern a voulu que soient animées en profondeur les interférences sonores de son oeuvre.

8 Il est en effet, dans son intégralité, symétriquement articulé autour d'un LA (celui du diapason). C'est donc sur les LA ou les RE/MIb de son texte que les affinités latentes devraient plus ou moins converger, en concordance avec le phrasé. Or, celui-ci y est totalement réfractaire. Certes, les RE/MIb y sont toujours émis avec l'intensité maximale $f f$ et même ils se manifestent, à la mesure 15, dans tout l'éclat de deux accords-types dont ils sont la note principale et avec l'appui de toutes leurs notes attractives. Mais, dans les mesures 6 et 21, ils se réduisent à des petites notes. Et le contexte n'autorise aucun aboutissement discursif sur les uns et les autres. Certes, deux LA successifs se retrouvent quatre fois à la même tessiture, mais en $p$ au milieu de sons $f$ ou $f f$ et sans être soulignés par le phrasé. Pourtant, si l'on en croit Stadlen qui y insiste (page II, par. 8 du texte allemand de son commentaire et page VI, par. 7 du texte anglais), Webern tenait absolument à ce que les LA en question ressortent chaque fois par un ralenti et un toucher très contrasté au milieu des sons très appuyés qui les précèdent et les suivent, et cela, malgré la vitesse du tempo, révélant ainsi, par ces incompatibilités avec l'écriture même de sa musique, à quel point il tenait à la logique fonctionnelle de l'affinité des sons qu'il mettait en oeuvre. 


\section{Cas d'affinités naturelles}

Répétons-le : chaque fois que, d'une manière ou d'une autre, Webern suspend le cours de son texte9 il prend soin de ponctuer la fin du membre de phrase interrompu, par la conjonction, avec ou sans reports d'octave, sur la note ultime, sur les notes ultimes, ou sur l'une de celles-ci, des affinités de tous ou d'une partie des sons à distance d'octave, de quinte, de quarte et de demi-ton de ces notes, en montant comme en descendant.

Dès les premières pages de l'oeuvre, on le constate, aux moments où des figures analogues de six sons se différencient par la présence ou l'absence d'un espacement entre elles : d'abord sur un MI,10 mesure 7, par propension d'un SI et d'un FA ; puis, mesure 10, sur un FA ${ }^{11}$ par celle d'un DO, d'un MI et d'un FA\# (cf. exemple 2). Aux arrêts suivants, le SI d'une part, le DO\# d'autre part échappent plus ou moins à cette dynamique, du fait de la proximité de leurs tritons respectifs, en raison de l'emprise de cet autre processus qui sera précisé : la logique de contraste (cf. exemple 3).

9 Seuls font exception, d'une part quatre des vingt-sept ritardando de l'ensemble : ceux des mesures 50-51 du ler mouvement et des mesures 34, 35, 39 et 49 du 3e mouvement, car ils procèdent en réalité de la logique d'opposition, en faisant contraste notamment avec les ritardando qui les suivent immédiatement, et d'autre part ceux des ralentis marqués par Webern d'un trait tremblé dans la nouvelle édition, qui figurent : l'un, mesure 14-15 du ler mouvement, dont il sera question à propos de cette même logique d'opposition, les autres, mesures $30-31$ et $32-33$, où ils ne constituent que des indications fugitives au milieu du jeu ininterrompu de triples croches.

10 Souligné, dans la nouvelle édition, par l'adjonction d'un cercle, et commenté pages III, par. 4 et VI, par. 4 de son "Introduction".

11 Souligné par un arrêt et l'adjonction d'un cercle, et commenté pages III, par. 4 et 10 et VI, par. 4 et 10. 
Après un passage où l'on constatera alors que prédomine cette même sorte de logique, une propension identique fait souligner, par le triton RE-SOL\#, l'arrêt sur le LA ${ }^{12}$ de la mesure 34 , et, au changement de figures qui s'annonce à la mesure 36 , intensifie, par les deux sons MI et $\mathrm{SIb}$, à la fois le SI et le MIb de cette mesure ${ }^{13}$ (cf. exemple 4).

Quant aux mesures 37 à 54 qui terminent le mouvement, elles constituent une transposition à la tierce majeure des 18 premières mesures et en reproduisent donc les convergences d'affinité, mais en en augmentant l'efficacité, comme on le constatera à propos des renforcements (cf. exemple 7).

Dans le troisième mouvement, ces propensions fonctionnelles procèdent davantage de la dialectique d'opposition. Mais leurs conjonctions attractives persistent, et notamment aux moments que le texte souligne le mieux, comme au long arrêt qui suit la mesure $43^{14}$ ou au point d'orgue de la mesure $55^{15}$ (cf. exemple 5).

Ces sortes de convergences ponctuent, en particulier, les nombreux ritardando du texte, à l'exception seulement de quatre qui, par leur contraste avec ceux qui les suivent immédiatement, relèvent de la logique d'opposition. 16

12 Souligné par l'adjonction d'un trait tremblé de ralentissement.

13 Souligné par l'adjonction d'un ralentissement et d'un point d'orgue.

14 Souligné par l'adjonction d'un point d'orgue, et commenté pages IV, par. 3 et VII, par. 3.

15 Souligné par l'adjonction d'un crescendo, et commenté pages 4 , par. 4 et VII, par. 4.

16 Voir note 9. 
En outre, qu'il s'agisse ou non des propensions en question, ceux des sons où la musique suspend plus ou moins son cours se trouvent fréquemment reliés entre eux par des liens d'affinité naturelle de quinte, de quarte ou de demi-ton. ${ }^{17}$

\section{Les renforcements d'affinité}

Toutes les fois qu'il fait réapparaître un ensemble de figures sous la forme d'une répétition, d'une transposition ou d'un renversement, au lieu d'observer une rigoureuse correspondance, Webern procède à des reports d'octaves ou à des déplacements qui, sans la moindre exception, viennent accroître le potentiel d'aboutissement de l'ensemble obtenu.

Ainsi, le passage qui occupe les mesures 8 à 10 du premier mouvement se termine, nous l'avons mentionné, en prenant appui sur le FA ultime (cf. exemple 2). Mais, en en reproduisant les sons dans les mesures 16 à 18 , Webern a isolé les six derniers par un silence, mettant par là mieux en évidence d'une part le DO, le MI et le FA\# qui concourent à l'affirmation du FA, 18 et, d'autre part, le DO\# qui, à l'arrêt ainsi provoqué, devient capable d'intensifier le FA\# de l'accord final'19 (cf. exemple 3).

De même dans les mesures 37 à 46 de ce mouvement, en transposant d'une tierce majeure ascendante les dix premières mesures, dont nous venons de rappeler l'aboutissement fonctionnel sur un FA, Webern

17 Points d'appui fonctionnels : mesures 7 à 10, 18 à 21, 23 à 29 et 43 à 46 du ler mouvement, et 24 à 33, 41 à 44,52 à 55 et 62 à 65 du 3e mouvement. Autres interruptions du texte : mesures 2 à 4, 17 à 18, 38 à 40, 46 à 51, 53 à 54 du ler mouvement, et 2 à 5,5 à 8,12 à 14,24 à 33, et 43 à 52 du $3 e$ mouvement.

Souligné par l'adjonction d'un cercle.

19 Commenté pages III, par. 10 et VII, par. 10 . 
prend soin, dans la mesure 46, d'augmenter le potentiel du LA, ${ }^{20}$ qui est l'homologue de ce FA, en rapprochant les notes attractives par des reports d'octaves (cf. exemple 6).

Les figures suivantes constituent le renversement intégral de celles des mesures 11 à 18, dont on sait qu'elles se stabilisent aussi sur un FA (cf. exemple 3). Du RE de la mesure 54, homologue de ce FA, Webern s'applique, par des reports d'octaves, à rapprocher les notes qui lui sont attractives : SOL, homologue du DO, et MIb, homologue du MI. Mais, en lui adjoignant son triton SOL\#, il le désagrège, et le fait participer à un accord-type de DO\#-RE-SOL\#-DO\#. Et c'est en définitive ce DO\#,21 homologue du FA\# de la mesure 18, qui, par la prépondérance ainsi acquise de son potentiel d'aboutissement, vient mettre un point final à ce premier mouvement. 22

Il en est de même des mesure 32 et 33 du troisième mouvement, où se trouvent reproduits les sons des mesures 28 et 29 ; ceux-ci sont toutefois resserrés de telle sorte que le rapprochement ainsi obtenu des affinités

20 Souligné par l'adjonction d'un cercle et d'un ralenti, et commenté pages III, par. 4 et VI, par. 4.

21 Souligné par l'adjonction d'un cercle.

22 Au lieu de placer à la portée inférieure de la mesure 54 l'accord final RE-DO\#, homologue du FA-FA\# de la mesure 18, Webern l'a hissé au sommet de la portée supérieure, en lui adjoignant un SOL\# qui le transforme en un accord-type de DO\#. Par remontée d'une octave, il a mis en évidence deux des notes attractives de ce DO\# (le SOL\# de la mesure 51, homologue du SI de la mesure 15, et le FA\# de la mesure 53, homologue du DO\# de la mesure 17). En outre, afin que le potentiel ainsi accru du DO\# ne soit pas contredit au triton par le SOL de la mesure 53, homologue du DO de la mesure 17, il a rapproché de ce SOL, en le haussant d'une octave, le MIb, homologue du MI, ce qui tend à les faire se résoudre l'un et l'autre sur les sons RE et SOL\# de l'accord-type ultime dont ce DO\# est la tonique. Toutefois, sera signalée, en conclusion, une autre interprétation possible de cette fin. 
vient très opportunément favoriser le potentiel d'aboutissement du MIb ultime, au moment précis de l'apparition de figures nouvelles.

Enfin, à la dernière page de l'oeuvre, le passage qui va du MI naturel de la mesure 59 à l'accord de la mesure 61 est transposé un demi-ton au-dessous, dans l'ensemble ultime qui commence au MIb de la mesure 64. Dans la mesure 60, le FA\# se trouvait déjà intensifié, à la fois comme note principale d'un accord-type ${ }^{23}$ et par mouvement direct d'un FA. Or, dans l'accord-type de la mesure 65, Webern s'est complu à porter à son maximum le potentiel du FA, homologue de ce FA\#, en le faisant précéder de l'accord non transposé de la mesure 59, dont le SIb se trouve ainsi placé en évidence, à distance juste de quarte de ce FA (cf. exemple 8).

Il en résulte cette constatation, qui peut être capitale pour l'exécution : ce FA est la seule de toutes les notes de ces Variations à bénéficier, au moment où la pièce fin, de la totalité de ses notes attractives, données dans la même tessiture. ${ }^{24}$

\section{Logique d'opposition}

En opposant l'instable au stable, dans la confrontation de ces deux attitudes contraires que constituent, d'une part la précarité, l'incertitude, l'irrésolution de figures dépourvues de convergences efficaces d'affinités, et d'autre part, la fermeté, l'assurance, l'affirmation de celles que le potentiel d'aboutissement de leur fin stabilise sur leur propre centre de gravité, Webern a adapté exactement à son oeuvre cette dynamique traditionnelle qui animait déjà l'alternance des fonctions de dominante et de tonique.

23 Souligné par l'adjonction d'une liaison et d'un point d'orgue.

24 Ce FA est en outre note principale d'un accord-type, que Webern lui-même a tenu à ériger en aboutissement d'un enchaînement où le SOL va vers DO et le MI vers FA, en le faisant précéder du mot "anschliessen". 
On l'a constaté dès les mesures 15 et 16 du premier mouvement, dont la précarité fait contraste avec l'appui que la mesure suivante prend sur le FA de la mesure $18^{25}$ (cf. exemple 3).

Mais c'est au plus spectaculaire de ces moyens discursifs qu'il recourt aussitôt après, et par trois fois, par la stabilisation immédiate du même son préalablement déséquilibré.

Dans l'accord FA\#-FA qui, au ritardando de la mesure 20, tend passagèrement vers le $\mathrm{FA}$, le $\mathrm{FA \#} \mathrm{se} \mathrm{trouve} \mathrm{déstabilisé} \mathrm{par} \mathrm{la} \mathrm{proximité} \mathrm{im-}$ médiate de son propre triton DO, alors que, à la mesure suivante, son exacte répétition, ${ }^{26}$ à la même tessiture, s'affirme hautement sous l'impulsion redoublée de deux DO\# et de deux SOL. Et le MIb de la mesure 23, ainsi que de SI de la mesure 29 bénéficient à leur tour de ce même pouvoir expressif de stabilisation (cf. exemple 9).

Quant au troisième mouvement, c'est la logique de contraste qui en anime l'intégralité. Après un début ponctué de l'alternance signifiante de figures solidement axées sur le centre de gravité de leurs affinités, ${ }^{27}$ parmi des figures dépourvues de tels appuis, Webern inaugure une nouvelle variante des moyens d'opposition, en procédant, tout au long

Souligné par l'adjonction d'un cercle.

26

Souligné par l'adjonction d'un arc vertical d'arrêt, comme le MIb de la mesure 23, et le SI de la mesure 29. Le MIb bénéficie en outre de l'adjonction d'un trait d'affinité. L'ensemble est commenté pages III, par. 4 et VI, par. 4.

27

Le SOL de la 3e mesure par DO et FA\#, le SI de la $8 \mathrm{e}$ mesure par FA et DO, le MIb de la 12e mesure par SIb et RE (dont Webern a voulu préciser l'impact en lui adjoignant un quasi vibrato qui le prolonge), le LA de la 14e mesure par LAb et SIb, le MIb de la 15e mesure par MI et SOL\#, les SOL\# de la $17 \mathrm{e}$ et de la 19e mesure par SOL et LA d'abord, puis par MIb, SOL et LA, et enfin le FA\# de la 21e mesure par DO\#, SOL et FA et le DO\# de la 23e mesure par FA\#, DO et RE. 
des dix mesures qui suivent l'arrêt de la mesure 43, à une augmentation progressive des contrastes.

Au SOL 28 de cette mesure 43, qu'affirment en $f f$ un SOL et un LA, succède l'incertitude de figures évasives, mais dont les propensions s'affirment peu à peu. ${ }^{29} \mathrm{Et}$ cette progression aboutit, au point d'orgue de la mesure 55, sur un sf qui, sous l'impulsion directe d'un DO et d'un MI, retentit en molto $f f$ comme un point d'exclamation, dans toute la plénitude que lui valait déjà, à l'arrêt de la mesure 53, son annonce dans la même intensité et à la même tessiture, et dont les adjonctions de Stadlen attestent la fonction ${ }^{30}$ (cf. exemple 5). Enfin, ultime aspect de cette cinétique d'opposition, à ce SI tellement affirmé par les affinités comme par l'écrit, s'opposent aussitôt, en $p p$, des figures dont - à l'exception de celle de la mesure 65 dont nous avons constaté l'irréfutable fixation - il est malaisé de déceler les centres de gravité, et qui semblent se dissoudre dans l'évanescence.

\section{Conclusions}

Ce n'est même pas par intuition, mais c'est indéniablement de propos délibéré que Webern, mettant à profit l'enseignement de Schoenberg sur "cet équilibre de toutes les forces de tension possibles" que les sons doivent à l'impulsion originelle de leurs "plus courts chemins", a si exactement imprégné les austères rigueurs de la technique sérielle du

Rappelons qu'il est souligné par l'adjonction d'un point d'orgue, et commenté pages IV, par. 3 et VII, par. 3.

Mesure 50 sur un SOL par un SOL\#, mesure 52 d'abord sur un RE par un DO\# et un MIb, puis sur un SIb qu'imposent les deux sons du triton SI-FA, car on peut admettre qu'il est préservé contre le pouvoir dissolvant du MI qui précède, par la double résolution de celui-ci sur le FA et le SI.

Souligné par l'adjonction d'un crescendo, et commenté pages IV, par. 4 et VII, par. 4. 
flux énergétique que les hauteurs reçoivent des affinités naturelles d'octave, de quinte, de quarte et de demi-ton.

A l'interprète qui sait en prendre assez conscience pour en faire siennes les pulsions vitales, il sera peut-être donné de magnifier ces Variations bien au-delà des signes et des adjonctions de leur texte, selon cette logique immanente par quoi Webern s'était plu à en transcender la lettre pour en vivifier l'esprit.

Ces tensions se restreignent aux hauteurs, et à celles-là seules qui relèvent d'échelles tempérées capables des trois premiers harmoniques. Mais leur prégnance y peut être alors sans limite, et l'oeuvre la plus réfractaire aux investigations usuelles ne résiste pas au test de leurs analyses.

Les affinités naturelles y peuvent déceler non seulement le jeu des cadences d'attraction, ${ }^{31}$ mais encore les points d'appui du phrasé ${ }^{32}$ et

31 Cf. in Costère 1962 : Debussy, Feuilles mortes, p. 160 ; Scriabine, $7 e$ Sonate pour piano, p. 158 ; Schoenberg, fin du Pierrot lunaire, p. 171, et début de la Fantaisie opus 47, p. 125 ; Webern, 2e Cantate, p. 174 ; Olivier Messiaen, Vision de l'Amen, p. 26.

Cf. Costère, ibidem : Sur le son ou l'accord ultime : Schoenberg, la lère des Pièces pour piano opus 11, p. III, 5e des Pièces pour piano opus 19, p. 109-110, die Mondfleck, p. 74, et fin des $2 \mathrm{e}$ et 3e parties du Pierrot lunaire, p. 169 à 171, et Fantaisie opus 47, p. 130 ; Bartók, ler mouvement de la Sonate pour piano, p. 114, 99e Mikrokosmos, p. 113 ; Webern, 2e des Mélodies opus 3, p. 77, Das Augenlicht, p. 81, lère partie de la lère Cantate, p. 75, dernière partie de la $2 e$ Cantate, p. 81 et 176 ; Honegger, Pacific 231, p. 25 ; Jolivet, 2 e mouvement de la lère Sonate pour piano, p. 154, et 5e des Danses rituelles, p. 112 ; Messiaen, 6e et 7e des Préludes pour piano, p. 109 et 161, et Modes de Valeurs et d'Intensités, p. 110 ; Boulez, lère Sonate pour piano, p. 69, et 3e mouvement de la $2 e$ Sonate pour piano, p. 76 ; Stockhausen, Fünf Zeitmasse, p. 77. Dans le cours de l'oeuvre : Schoenberg, Fantaisie opus 47, p. 125 ; Webern, 2e Cantate, p. 82, 174 et 175 ; Messiaen, Vision de l'Amen, p. 26 ; Stockhausen, Fünf Zeitmasse, p. 68. 
même la continuité d'une tonique, ${ }^{33}$ ainsi que tous les aspects d'une dialectique de contraste. ${ }^{34}$ Elles en définissent même le style, car, face au désordre intrinsèque des harmonies sans loi qu'elles détectent, elles départagent l'intégralité des harmonies qu'elles animent, en faisant le départ entre harmonies de style naturel, ${ }^{35}$ où la gravitation est souterraine, et celles de style modal, ${ }^{36}$ qui parviennent à s'articuler autour de centres de gravité secondaires dont des renforcement appropriés ont fait prédominer le potentiel.

Mais ce pouvoir des affinités naturelles de faire signifier les hauteurs ne relève pas seulement du quantitatif. Leur tension varie en fonction de la proximité et de l'importance de notes capables d'attraction, dont chacun peut éprouver différemment l'incidence.

Ainsi, en dépit de la fonction de convergence des accords-types, il est loisible à l'interprète que ne satisfait point la prédominance fonctionnelle de l'accord-type qui clôt le premier mouvement des Variations Opus 27, d'exprimer par son jeu l'éventuelle incertitude de cette fin -

33 Costère, ibidem : Schoenberg, Menuet de l'Opus 25, p. 84, 85 ; Jolivet, 4e des Mana, p. 111.

34 Costère, ibidem : Scriabine, 7e Sonate pour piano, p. 123 ; Schoenberg, 2 e des Pièces pour piano opus 11, p. 80 ; Pièce pour piano opus $33 a$, p. 81, et Fantaisie opus 47, p. 69 ; Webern, Mélodie opus 12, 1ère des Mélodies opus 13, p. 79 et $2 e$ Cantate, p. 82 ; Jolivet, lère Sonate pour piano, p. 121 ; Messiaen, 2e et 6e des Préludes pour piano, p. 121, 122 ; Neumes rythmiques, p. 123.

35 Je l'avais nommé "style cardinal" in Costère 1954. Il s'agit ici de tous les exemples cités dans les notes 31 à 34 .

Cf. Costère 1962. Sont de style modal la totalité de l'Hommage à Rameau de Debussy (Costère, ibid.. : 145 à 149) et des passages des oeuvres suivantes : Scriabine, 7e Sonate pour piano, p. 157, 165 ; Bartók, Sonate pour piano, p. 157, 162 ; Stravinsky, Trois mouvements de Petrouchka, p. 155, et Concertino pour quatuor à cordes, p. 163 ; Jolivet, lère Sonate pour piano, p. 155, 159, 166 ; Messiaen, 6e des Préludes pour piano, p. 163. 
dont le letzter Seufzer de Peter Stadlen peut autoriser l'évanescence car il peut estimer que le SOL qui précède n'a pas épuisé, en se résolvant sur le RE et le SOL\# ultimes, son emprise dissolvante de triton sur le DO\# final (cf. exemple 7).

Quant aux dernières mesures de l'oeuvre, elles demeurent sans force dans leur nuance $p p$ qui s'estompe jusqu'au $p p p$. Certes, l'on pourrait consacrer l'aboutissement, sur le LA final, du RE qui le précède et du SIb qui le joint, en tant que terme ultime de l'oeuvre entière. Mais, rappelons-le, Webern a non seulement pris soin de valoriser, comme centre de gravité des avant-dernières mesures, le FA de la mesure 65, en lui adjoignant - seul de tous les points d'appui fonctionnels du texte - la totalité de ses notes attractives, mais encore, il a tenu à souligner par le mot anschliessen, dans l'exemplaire conservé par Stadlen, l'arrivée sur l'accord où ce FA prédomine. Une prépondérance si intentionnelle peut d'autant moins s'éluder que, par sa ténuité, la dernière mesure semble se résoudre en un point de suspension ... (cf. exemple 8).

Quoi qu'il en soit de ces appréciations relevant de l'intuition musicale, ces quelques analyses fonctionnelles témoignent d'une telle authenticité des agencements latents qui peuvent animer les sons, qu'il est difficile de ne pas en rapprocher le dispostif des analyses structurales.

Pour Claude Lévi-Strauss, à qui j'en avais demandé la définition, "le Structuralisme s'attache à dégager les lois universelles en quoi consiste l'activité inconsciente de l'esprit". ${ }^{37}$ Les lois universelles ... : celles des mouvements premiers de l'acoustique ; ... en quoi consiste ... : notre organe pharyngo-buccal nous rend ces mouvements consubstantiels ; ... l'activité inconsciente de l'esprit : notre entendement perçoit ces mouvements comme une relation d'affinité entre les sons qu'ils unissent.

Grâce à ces moyens d'un structuralisme en quelque sorte viscéral, l'oeuvre qui, par acte conscient ou non, en détient les pouvoirs, si

37 Lettre du 16 mars 1982 à Edmond Costère. 
ésotérique qu'elle paraisse, recèle au tréfonds d'elle-même l'en-soi d'une dynamique naturelle, que l'interprète ne peut plus taire s'il en a ressenti la logique, et dont la découverte peut conduire le musicologue, abusé par des exégèses déficientes, ${ }^{38}$ à en clarifier le sens.

Mais dans l'appréciation de l'incidence des affinités réciproques d'octave, de quinte, de quarte et de glissement, la sensibilité de l'un et de l'autre reste souveraine. Et c'est le tempérament musical de chacun qui, vraiment, importe, et l'emporte.

38 Cf. Leibowitz 1947 et 1949, Falk 1959, et Rufer 1952. La formule de Schoenberg définissant la série comme une suite de "douze sons indépendants" ne visait que leur indéniable indépendance à l'égard de la tonique fondamentale du diatonisme. Mais prise au pied de la lettre, elle s'est perpétuée dans l'esprit de cette définition du dodécaphonisme exposée dans un numéro spécial sur la musique de Science et Vie : "La musique de douze sons de Schoenberg et de ses disciples, de ce que l'on appelle l'Ecole de Vienne, accorde aux douze notes, les douze demi-tons de la gamme chromatique tempérée, une égale dignité ; le compositeur ne doit conférer à aucune d'elles un rôle particulier ; aucune ne doit exercer d'attraction sur une autre" (1962 : 78). Pour le lecteur de langue française, les traductions, trop tardives hélas, des écrits de Schoenberg vont enfin donner le coup de grâce à ce contresens affligeant qui a tout faussé. 


\section{RÉFÉRENCES}

COSTËRE, E.

1954: Lois et styles des harmonies musicales. Paris : Presses Universitaires de France.

1962: Mort ou transfigurations de l'harmonie. Paris : Presses Universitaires de France.

\section{LEIBOWITZ, $R$.}

1949: Introduction à la musique de douze sons. Paris : L'Arche.

\section{SCHOENBERG, A.}

1977: Le Style et l'Idée. Paris : Buchet-Chastel.

1983: Traité d'harmonie. Paris : Lattès. Traduction et présentation de Gérard Gubisch.

\section{WEBERN, A.}

1980: Chemins vers la nouvelle musique. Paris : Lattès. 

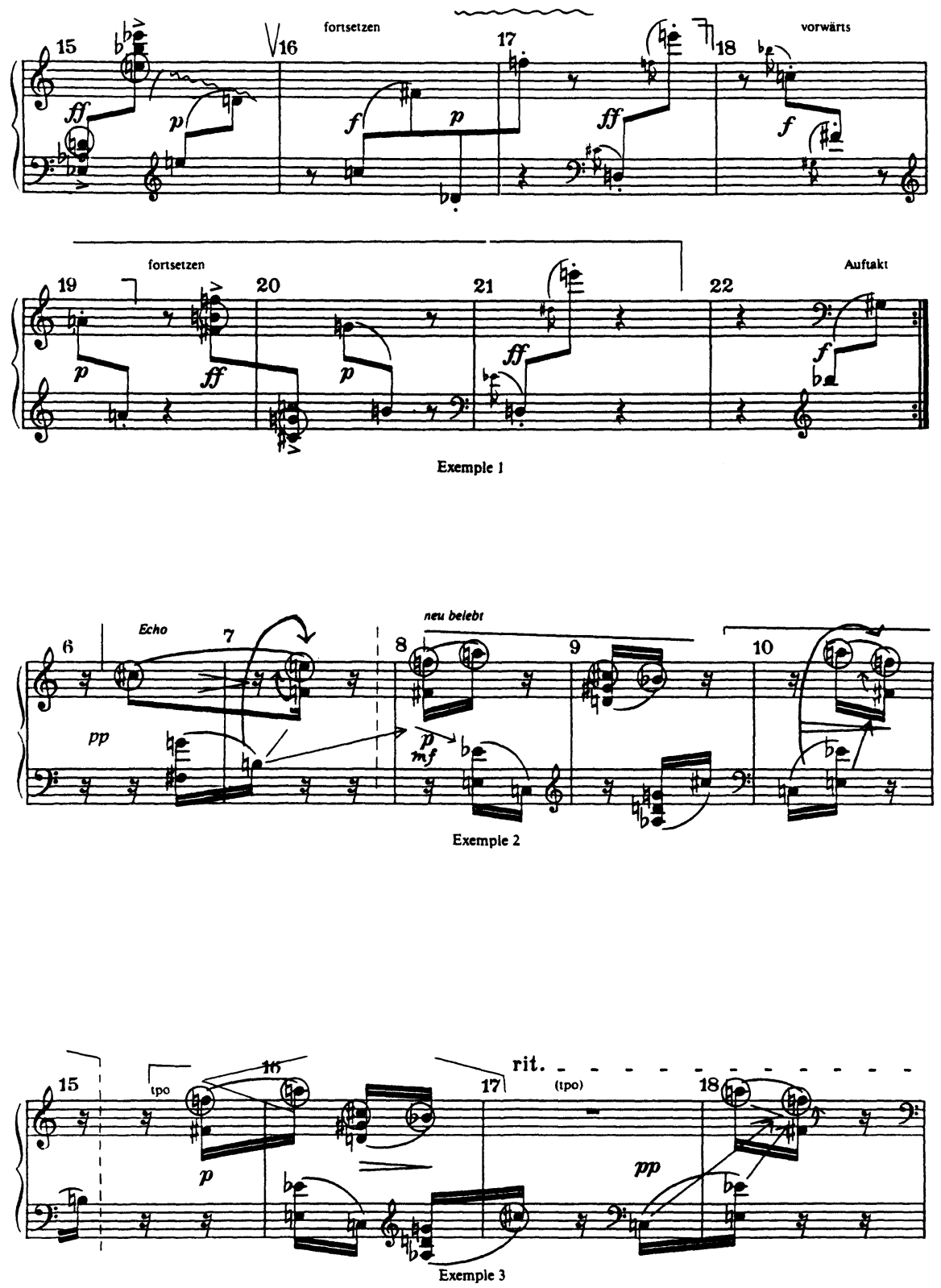

N.B. Les fièches en traits fins sont de Stadlen dans la nouvelle édition des variations op. 27. 

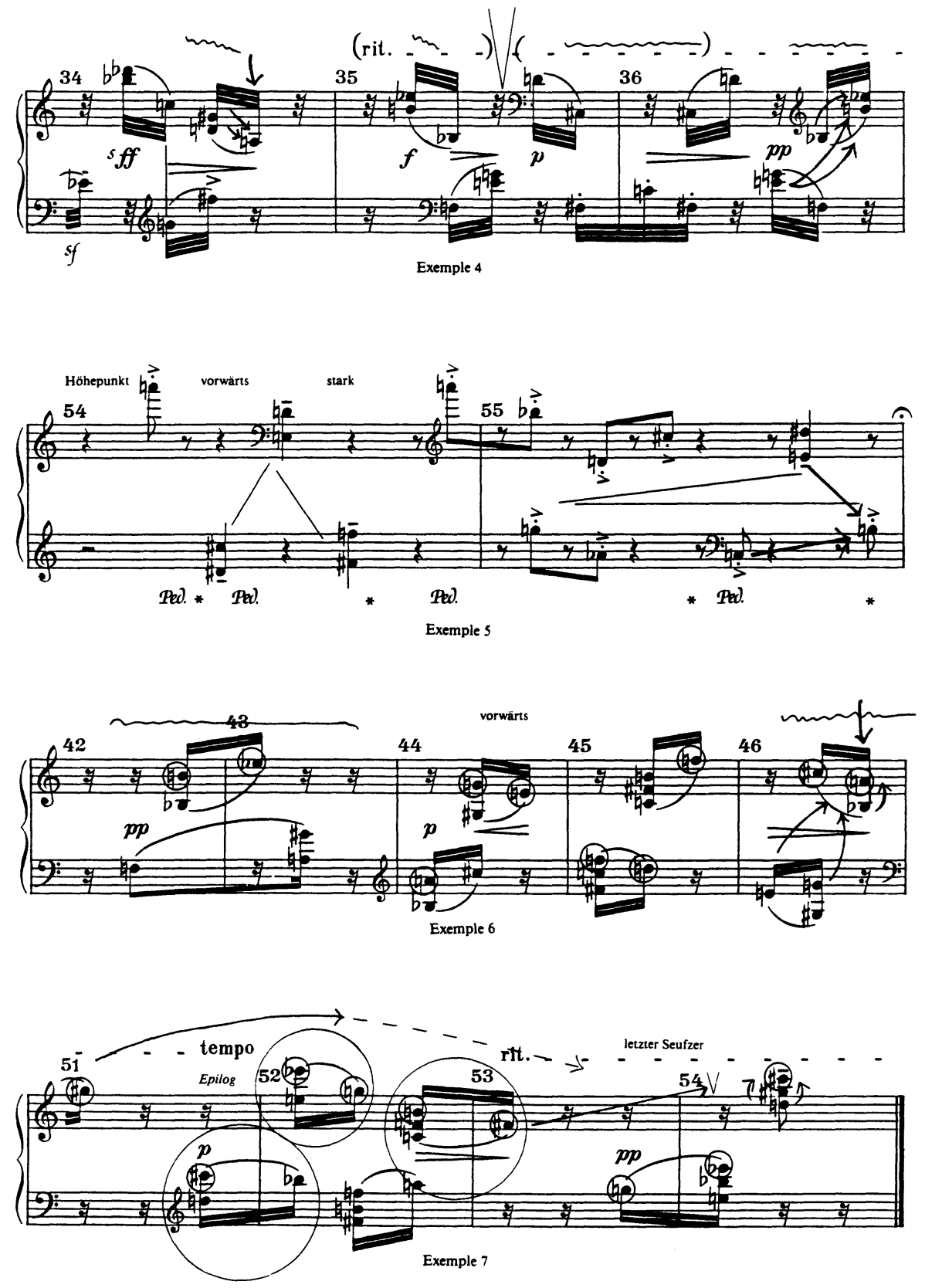

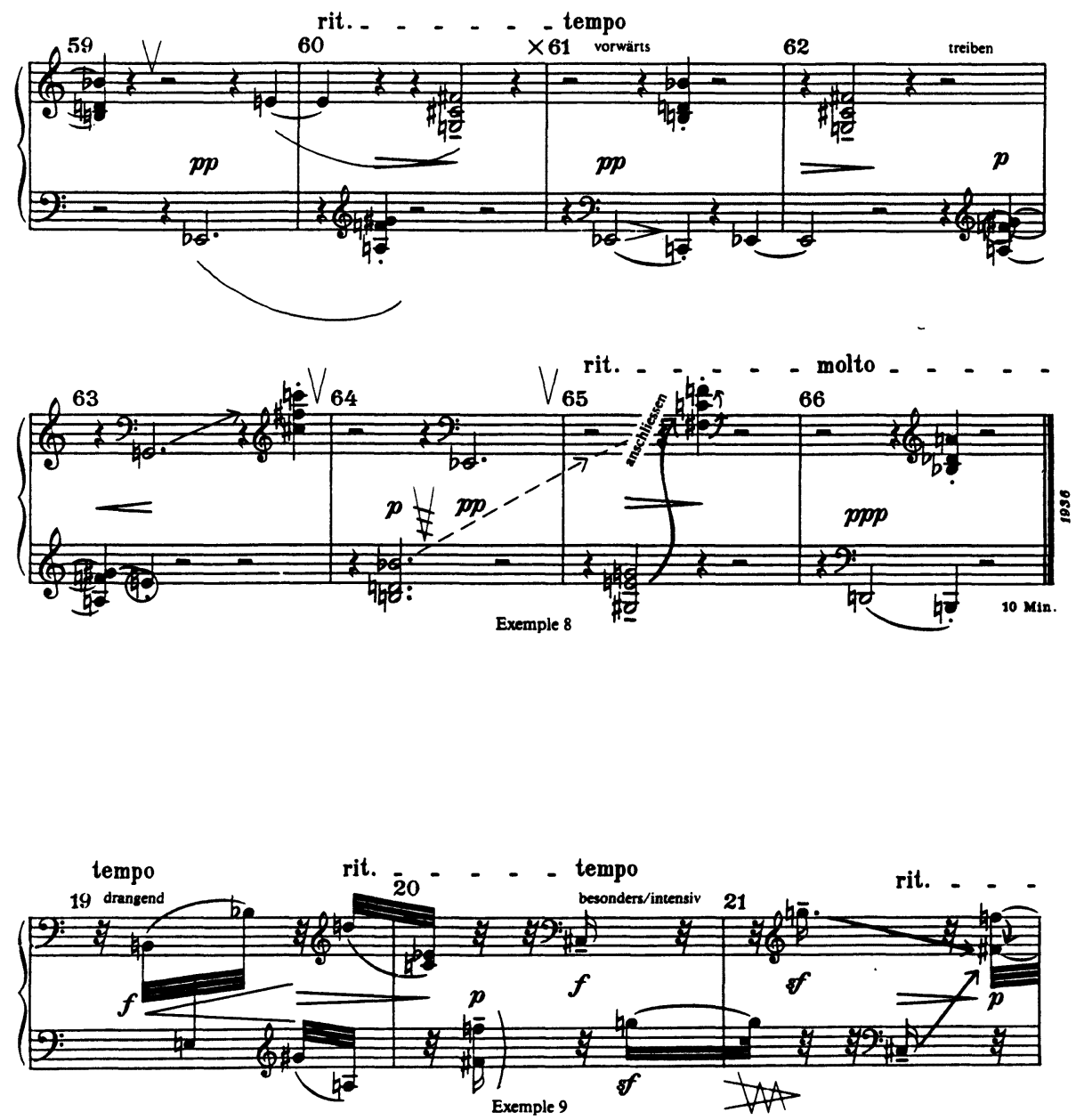

“Copyright ${ }^{\odot} 1937$ by Universal Edition" "Reprinted by Permission" 